\title{
DIE ENTWICKLUNG DES ISLAMISCHEN FINANZWESENS IN DER GOLFREGION
}

\author{
Heiko SCHUSS
}

\section{Zusammenfassung}

Dieser Artikel beschreibt die Entwicklung des Islamischen Finanzwesens in der Golfregion und sucht zu erklären, wie es dieser Region gelang, ihre führende Rolle in dieser Industrie zu steigern. Er nutzt hierzu die einschlägige Sekundärliteratur und die Statistiken des Islamic Financial Services Board (IFSB). Der Artikel behandelt sowohl die Staaten des Golf-Kooperationsrats als auch Iran. Diese Länder verfügen weltweit über die Mehrheit der islamischen Finanzanlagen. Die Golfregion spielt eine zentrale Rolle im Islamischen Finanzwesen und dieses verbindet die Golfregion mit anderen Weltregionen. Diese beherrschende Rolle der Golfregion beruht auf den Präferenzen ihrer Bevölkerung für shari'a-konforme Produkte, ihre Finanzkraft, die politische Unterstützung der Regierungen und die Internationalisierungsbestrebungen ihrer islamischen Finanzinstitute. Der Artikel beleuchtet auch die Unterschiede innerhalb der Region.

Schlagwörter: Islamisches Finanzwesen, Islamische Banken, Sukuk, Golfregion, Golf-Kooperationsrat, Iran.

\section{淡淡 \\ KÖRFEZ BÖLGESINNDE İSLAMİ FINAANSIN GELİŞIMİ \\ Özet}

Bu makale, Körfez bölgesinde İslami finansın gelişimini anlatmak ve bölgenin bu endüstrideki öncü rolünü nasıl geliştirdiğini açıklamayı amaçlamaktadır. Makalede ilgili ikincil literatür ve İslami Finansal Hizmetler Kurulu'nun (Islamic Financial Services Board, IFSB) istatistikleri kullanılmış ve yorumlanmıştır. Bu makale hem Körfez İşbirliği Konseyi (Gulf Cooperation Council, GCC)) ülkelerini hem de İran'ı içermektedir. Bu ülkeler, dünya genelindeki İslami finansal varlıklarının önemli bir bölümüne sahiptir. Körfez bölgesi, İslami finans alanında merkezi bir rol oynamakta ve İslami finans, Körfez bölgesini diğer dünya bölgelerine bağlamaktadır. Körfez bölgesinin bu baskın rolü, nüfusunun şer'i hükümlere uygun ürünleri tercih etmesinden, mali gücünden, hükümetlerin politik desteğinden ve İslami finans kurumlarının uluslararasılaşma çabalarından kaynaklanmaktadır. Makale, bölge içi farklılıklara da ışık tutacaktır.

Anahtar Kelimeler: İslami Finans, İslami Bankacılık, Sukuk, Körfez Bölgesi, GCC, İran.

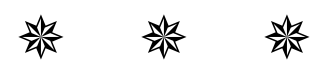

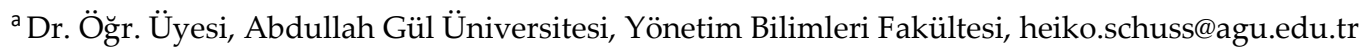




\section{THE DEVELOPMENT OF ISLAMIC FINANCE IN THE GULF REGION}

\section{Abstract}

The article aims at describing the development of Islamic finance in the Gulf region and to explain how the region was able to increase its leading role in this industry. It uses and interprets relevant secondary literature and the statistics of the Islamic Financial Services Board (IFSB). This article includes both the countries of the Gulf Cooperation Council (GCC) and Iran. These countries own the majority of the Islamic financial assets world-wide. The Gulf region plays a central role in Islamic finance and Islamic finance connects the Gulf region with other world regions. This dominating role of the Gulf region is caused by the preference of its population for shari'a-compliant products, its financial strength, the political support of the governments and the internationalization efforts of its Islamic financial institutes. The article will also throw light on the differences within the region.

Key Words: Islamic Finance, Islamic Banking, Sukuk, Gulf Region, GCC, Iran.

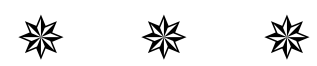

\section{Einführung}

Während islamische Finanzinstitute zu ihren Anfängen in den 1960er und 1970er Jahren von westlichen Beobachtern eher als eine kulturelle Randerscheinung denn als Ausdruck eines tragfähigen Geschäftsmodells betrachtet wurden, hat sich das islamische Finanzwesen zu einem einträglichen Geschäft entwickelt, welches weltweit Beachtung findet. Da die Interpretation des islamischen ribaVerbotes als eines Verbotes jeglicher Zinsen eine der wichtigen Grundlagen des islamischen Finanzwesens bildet, könnte man vermuten, dass es in allen Ländern mit muslimischer Mehrheitsbevölkerung gleichermaßen Zuspruch findet. Dem ist aber keineswegs so. Während in den meisten Weltregionen das islamische Finanzwesen eine nachgeordnete Stellung einnimmt bzw. dort nur in einzelnen Ländern wie Malaysia und Sudan eine wichtige Rolle spielt, ist das islamische Finanzwesen in der gesamten arabisch-persischen Golfregion von herausragender Bedeutung. Dieser Artikel möchte daher die Entwicklung des islamischen Finanzwesens in der Region des arabisch-persischen Golfs beschreiben und aufzeigen, wie die Golfregion ihre führende Rolle in dieser Industrie ausbauen konnte. Er nutzt hierzu die einschlägige Sekundärliteratur und die Statistiken des Islamic Financial Services Board (IFSB). Hierbei wird sowohl auf die Staaten des Gulf Cooperation Council (GCC) als auch auf Iran eingegangen. Auf diese Länder konzentriert sich die Mehrheit der Anlagen des islamischen Finanzwesens weltweit. Es kann dies als ein Charakteristikum der Region angesehen werden, durch welches sich die Golfregion von anderen Weltregionen unterscheidet und das von den Geschäftspartnern aus muslimischen wie nicht-muslimischen Regionen berücksichtigt werden muss. Das islamische Finanzwesen ist daher nicht nur ein unterscheidendes Merkmal der Golfregion, es verbindet auch diese Region mit Ländern und Finanzzentren anderer Regionen der islamischen und westlichen Welt. Obwohl das islamische Finanzwesen gegenwärtig in allen Ländern der Golfregion beheimatet ist, bestehen wichtige Unterschiede in der Umsetzung und im zeitlichen Verlauf. Auf diese bedeutenden Unterschiede innerhalb der Golfregion soll im Folgenden auch eingegangen werden. 


\section{Beginn des Islamischen Finanzwesens in der Golfregion}

Während die Konzepte für eine moderne islamische Wirtschaft in Südasien entwickelt und die ersten Pilotprojekte mit islamischen Finanzinstituten in Ägypten, Pakistan und Malaysia unternommen worden waren, wurden die ersten islamischen Geschäftsbanken in den arabischen Golfstaaten in den 1970er Jahren gegründet. Die Motivation hierfür kann einerseits in der Religiosität und der vorherrschenden Interpretation des riba-Verbots ${ }^{1}$ als eines Verbots jeglicher Zinsen in der Region gesehen werden und andererseits in der Tatsache, dass das moderne konventionelle Finanzwesen im Gegensatz zu anderen Ländern des Nahen Ostens später Fuß in den arabischen Ländern des Golfs gefasst hatte. Häufig wurden die Finanztransaktionen noch von Geldwechslern und Kaufleuten betrieben. Als Beispiel hierfür kann die Beschreibung Rodney Wilsons (1983) der Geldwechsler von Dschidda noch Anfang der 1980er Jahre dienen. Diese waren räumlich im Markt konzentriert direkt neben den Goldhändlern, welche für die Bewertung von Schmuck und Edelmetallen herangezogen werden konnten. Ein Hauptgeschäft war der Wechsel der von den Pilgern mitgebrachten Devisen. Aber sie nahmen auch Einlagen an, verliehen Geld und betrieben Warengeschäfte. Sie wurden als Familienunternehmen betrieben und konnten auf andere Familienmitglieder als sichere Kunden und potentielle Kapitalgeber zurückgreifen. Sie waren eng und langfristig mit der lokalen Gemeinschaft der Händler und Gewerbetreibenden verbunden. Zwischen den einzelnen Geldwechselunternehmen, die auch räumlich im Markt konzentriert waren, herrschte kein harscher Preiswettbewerb, sondern eher kooperative Beziehungen, wo ein Unternehmen kurzfristig Liquidität von anderen leihen konnte. Die Kenntnisse der lokalen Wirtschaft und Familien machten die Kreditvergabe und andere Geschäfte einfacher, da die Kreditwürdigkeit der Kunden bekannt war. Hierin bestand auch ein großer Vorteil gegenüber den bestehenden Geschäftsbanken. Geldwechsler vergaben Kredite mit einem Minimum an bürokratischem Aufwand und waren zeitlich wesentlich flexibler als die Banken. Einleger von Depositen erhielten zwar keine Vergütung, sie konnten aber sicher sein, dass sie ihr Geld zurückerhielten und dass sie zu bevorzugten Bedingungen Kredite erhalten würden. Kredite wurden wiederum nicht nur in Geld, sondern auch in Waren verliehen oder unter der Bedingung, die benötigten Waren bei einem bestimmten Händler zu kaufen. Hieraus wird deutlich, wie eng Geld- und Warengeschäfte miteinander verbunden waren. Die beschriebenen Transaktionen können auch alle als islamkonform ${ }^{2}$ bewertet werden. Später 1987 sollte das erfolgreichste dieser Geldwechselunternehmen Al-Rajhi eine Banklizenz erhalten und sich zur ersten islamischen Bank Saudi-Arabiens entwickeln (Wilson, 1983, S. 7-18; Wilson, 2009, S. 6).

Eine Wende in der Ausrichtung der zaghaften Anfänge des islamischen Finanzwesens brachte 1975 die Gründung der ersten islamischen Geschäftsbank, der Dubai Islamic Bank. Ihr Gründer war ein erfolgreicher Geschäftsmann mit guten Beziehungen zum Herrscherhaus Dubais (Kahf, 2005, S. 21). In den folgenden Jahren wurden mehr islamische Geschäftsbanken gegründet, so das Kuwait Finance House (1977) und die Bahrain Islamic Bank (1979). Zur gleichen Zeit wurden die ersten islamischen Investmentgesellschaften gegründet, z. B. die Islamic Investment Company Ltd. (1977) auf den Bahamas

\footnotetext{
${ }^{1}$ In diesem Artikel wird für die arabischen Fachbegriffe eine vereinfachte Umschrift benutzt, die sich an die Verwendung in englischsprachigen Publikationen zum islamischen Finanzwesen anlehnt.

${ }^{2}$ Islamkonform wird hier und später im Text im Sinne der Vertreter des islamischen Finanzwesens benutzt. Natürlich gibt es auch andere Interpretationen zentraler Begriffe - wie des riba-Verbots - im Islam, welche zu anderen Ergebnissen führen.
} 
und deren Tochter die Islamic Investment Company of the Gulf (1978) in Sharjah. Islamische Banken engagierten sich auch frühzeitig bei der Entwicklung islamischer Versicherungen, so gründete die Dubai Islamic Bank 1979 die Arab Islamic Insurance Company (Nienhaus, 1982, S. 249-253). Die Gründungen der ersten islamischen Banken verweisen auf enge Kooperationen zwischen den islamischen Bankern und den jeweiligen muslimischen Staaten und ihren Regierungen. So hielten die Staaten Dubai und Kuwait jeweils 10\% der Dubai Islamic Bank und der kuwaitische Staat 49\% am Kuwait Finance House (Wilson, 1983, S. 81).

An der Gründung islamischer Banken waren nicht nur religiös gesinnte Geschäftsleute, sondern auch Mitglieder der politischen Elite, z.B. des saudischen Königshauses, als Unternehmer beteiligt. Hier ist Prinz Muhammad al-Faisal zu nennen. Mit der Gründung islamischer Geschäftsbanken förderte er sowohl seine ökonomischen Interessen als auch den saudischen Einfluss im muslimischen Ausland. Ein bezeichnender Widerspruch ist, dass Saudi-Arabien über Jahre hinweg die Bezeichnung zinsfrei arbeitender Banken als islamische Banken im Inland nicht erlaubte, jedoch die Entwicklung und Verbreitung des islamischen Finanzwesens im Ausland förderte. Man war also bestrebt, die politökonomische Balance im eigenen Land nicht zu verändern (Kahf, 2005, S. 20-22; Wilson, 2009, S. 5-6).

Durch die Finanzminister der Mitgliedsstaaten der Organisation der Islamischen Konferenz wurde der Vertrag über die Islamic Development Bank verabschiedet, welcher 1975 in Kraft trat (Nienhaus, 1982, S. 242). Dies geschah auf Initiative von Algerien, Saudi-Arabien und Somalia. Mit dem Anstieg der Ölpreise 1973 standen den Ölstaaten große Mittel zur Verfügung, die sie nun auch außenpolitisch einsetzen wollten. Die Islamic Development Bank mit Sitz in Dschidda war hierfür ein Instrument, um Hilfsgelder an ärmere muslimische Staaten zu verteilen und hierdurch den Einfluss der Ölstaaten zu erhöhen. Sie finanzierte zunächst die Ölimporte muslimischer Staaten mit zinslosen Krediten. Die Islamic Development Bank sollte islamkonform arbeiten, wobei zur damaligen Zeit niemand eine klare Vorstellung davon hatte, wie dies praktisch umzusetzen sei. Interessant ist auch, dass die drei initiierenden Staaten bei sich selbst jahrelang keine islamischen Banken erlaubten. Es ging ihnen also nicht um eine konsequente islamische Wirtschaftspolitik, sondern eher um die Nutzung eines islamischen Images in der Außenpolitik (Kahf, 2005, S. 20-21).

Sowohl die Islamic Development Bank als auch die Dubai Islamic Bank ließen sich jeweils von islamischen Rechtsgelehrten beraten, sie besaßen aber noch kein shari'a-board im eigentlichen Sinne und es sollte noch Jahre dauern, bis sie ein shari'a-board einführten. Die Entwicklung islamkonformer Instrumente und Regeln des Finanzwesens war also zu Beginn mehr den Vorstellungen der Praktiker als den Rechtsgelehrten unterworfen. Einen wichtigen Schritt ging der saudische Prinz Muhammad al-Faisal mit der Einrichtung eines shari`a-boards für die Faisal Islamic Bank of Egypt (gegründet 1977). Die Bank benötigte sowohl das Vertrauen der potentiellen Kunden als auch des politischen Regimes. Aus diesem

| 450 | Grund suchte die Bank nicht die Zusammenarbeit mit den islamistischen Muslimbrüdern, sondern mit den ägyptischen Rechtsgelehrten. Zu diesem Zweck wurde ein shari'a-board der Bank eingerichtet, in dem angesehene ägyptische Rechtsgelehrte saßen, u.a. ein ehemaliger Großmufti Ägyptens. Die in den folgenden Jahren gegründeten islamischen Geschäftsbanken übernahmen alle die Einrichtung eines shari'a-boards. Das shari'a-board sollte fortan ein prägender Bestandteil islamischer Banken sein, 
welcher die Koalition zwischen islamischen Bankern und islamischen Rechtsgelehrten institutionalisierte (Kahf, 2005, S. 21-24).

Diese Pioniere entwickelten die Basisinstrumente des islamischen Finanzwesens und setzten diese in der Praxis ein. Das Modell der islamischen Geschäftsbanken beruhte hauptsächlich auf der Vergabe kurzfristiger Handelskredite durch murabaha Kontrakte (Wiederverkauf mit vorher vereinbartem Gewinnaufschlag), welche eine feste Gewinnmarge versprachen. Hierfür wurde diese traditionelle islamische Vertragsform neu interpretiert, was zu Beginn von verschiedenen Seiten kritisiert wurde, jedoch langfristig auf weite Zustimmung stieß (Wilson, 2009, S. 10-11). Es war ein unerlässlicher Schritt, der bei allen Unzulänglichkeiten bewies, dass es möglich war, Banken ohne Zinsen zu betreiben und auch auf privatwirtschaftlicher Ebene Gewinne zu erzielen. Dieser grundlegende Erfolg führte dazu, dass in mehr und mehr Ländern islamische Banken eröffnet wurden bzw. dass sich diese Länder für das islamische Bankwesen öffneten.

Wie bereits erwähnt war die politische Unterstützung durch die jeweilige Regierung ein wichtiger Erfolgsfaktor. Zunächst einmal war diese Unterstützung in dem Sinne unerlässlich, dass die Regierung zumindest die Gründung islamischer Finanzinstitute tolerierte bzw. erlaubte. Die arabischen Golfstaaten und ihre Regime konnten die Unterstützung islamischer Finanzinstitute für die Pflege ihres islamischen Images nutzen. Besonders interessant ist hierbei das differenzierte Vorgehen Saudi-Arabiens, das auf der internationalen Ebene die Gründung der Islamic Development Bank als Instrument einer eigenen Entwicklungspolitik betrieb, aber im eigenen Land das explizite Entstehen islamischer Banken über lange Zeit verhinderte (Henry, 2005, S. 108-109; Kahf, 2005, S. 20-21; Warde, 2000, S. 208).

Die arabischen Golfstaaten - mit Ausnahme Omans - förderten islamische Finanzinstitute, aber sie taten dies im Rahmen eines gemischten Finanzsystems, welches weiterhin durch die konventionellen Finanzinstitute geprägt war. Anders war dies in Iran nach der Islamischen Revolution 1979. Hier war der Anspruch, das gesamte gesellschaftliche System, somit auch die Wirtschaft, gemäß islamischen Regeln zu organisieren. In Iran wurde 1983 die gesamte Wirtschaft aus formaler Sicht zinsfrei umgestaltet (Brauksiepe, 1992, S. 1-3).

Ein Erfolgsfaktor, der vor allem in den 1970er Jahren eine Rolle spielte, war der Ölboom, welcher den Golfstaaten massive Gewinne und Deviseneinnahme einbrachte. Damit war die Grundlage für Investitionen im Inland und im Ausland gegeben. Zwar floss ein Großteil der Gelder, welche nicht im Inland absorbiert werden konnten, in konventionelle Finanzanlagen im Ausland. Jedoch war eben auch reichlich Kapital für eigene Projekte vorhanden. Wenn nur ein Bruchteil der Anleger in den Golfstaaten Wert auf islamkonforme Anlagen legte, so war dies verbunden mit dem neuen Reichtum ausreichend, um ein Geschäftsfeld für die neu entstehenden islamischen Geschäftsbanken zu bilden (Wilson, 1983, S. 80).

\section{Entwicklung in den 1980er und 1990er Jahren}

Seit den späten 1970er Jahren entwickelten die islamischen Finanzinstitute die Grundlagen für ihre Geschäftsmodelle. Traditionelle Vertragsformen, wie mudaraba (stille Gesellschaft), musharaka (Gesellschaft mit Gewinn- und Verlustbeteiligung), murabaha (Wiederverkauf mit vorher vereinbartem 
Gewinnaufschlag), ijara (Miete) und istisna' (Werklieferungsvertrag), wurden den modernen Bedürfnissen gemäß interpretiert und weiterentwickelt (Obaidullah, 2005, S. 52-93, S. 99-100). Hierfür war die Zusammenarbeit von Finanzexperten, islamischen Rechtsgelehrten und westlich geschulten Juristen notwendig. Es wurden auch die ersten Versionen islamkonformer Anleihen entwickelt, welche dann seit den 2000er Jahren als sukuk populär werden sollten.

In den GCC Staaten wurde die Entwicklung islamischer Banken dadurch begünstigt, dass in den 1990er Jahren die Gehaltszahlungen an Staatsbedienstete von Barzahlung auf Überweisungen auf Gehaltskonten umgestellt wurden. Dasselbe galt für andere staatliche Zahlungen an die Bürger. Da viele Bürger der GCC Staaten Zinszahlungen als riba betrachteten und scheuten, eröffneten diese ihre Konten bei islamischen Banken, was diesen eine breitere Kundenbasis brachte (Wilson, 2009, S. 12).

Die Entwicklung des islamischen Bankwesens im Iran beschritt einen grundsätzlich anderen Weg als die arabischen Nachbarländer. Nach der Islamischen Revolution 1979 wurde das Bankwesen verstaatlicht und sodann in zwei Schritten 1980 und 1983 zumindest formell vollständig islamisiert. Dies geschah aber nach Auffassung seiner Kritiker in einer oberflächlichen Weise, da sich die Profitraten und Bearbeitungsgebühren der neuen islamischen Banken nicht sehr von den Zinsen konventioneller Banken unterschieden. So wurden Zinsen zwischen den Banken weiterhin verwendet, da diese nun alle dem Staat gehörten und daher Verrechnungszinsen zwischen verschiedenen staatlichen Einheiten nicht als riba angesehen wurden (Brauksiepe, 1992, S. 2-3). Weiterhin setzte die Zentralbank untere Profitraten für verschiedene Investitionsarten und untere wie obere Grenzen für die Bearbeitungsgebühren fest. Der Staat kontrollierte auch über Kreditrationierungen die Investitionen in den verschiedenen Sektoren und begünstigte in den 1980er Jahren insbesondere den öffentlichen Sektor und den Bausektor. Da die Profitraten für die privaten Sparer niedrig und die Inflation hoch waren, kann auch von einer finanziellen Repression ausgegangen werden (Valibeigi, 1992, S. 56-65). Diese Art der Gestaltung des islamischen Finanzwesens verursachte also erhebliche Ineffizienzen.

Während dieser Jahrzehnte gründeten islamische Banken der Golfregion Joint-Ventures in anderen muslimischen Ländern und halfen, das Modell des islamischen Finanzwesens weiterzuverbreiten. Als Beispiel kann die Gründung der ersten islamkonformen Spezialfinanzinstitute (Al-Baraka Türk; Faisal Finance Institution, Kuwait-Turkish Evkaf Finance House) in der Türkei unter der Regierung Turgut Özals in den 1980er Jahren dienen, welche dabei helfen sollten, Investitionen aus den arabischen Golfstaaten ins Land zu holen (Henry, 1997, S. 123-126). Innerhalb der Golfregion entwickelte sich Bahrain zum Zentrum des islamischen Finanzwesens. Im Gegensatz zu anderen Golfstaaten erlaubte Bahrain die Tätigkeit ausländischer Banken. Hiermit zog es während des libanesischen Bürgerkriegs das Bankgeschäft aus Beirut an sich. Bahrain bemühte sich nicht nur ein Zentrum des konventionellen, sondern auch des islamischen Bankwesens zu werden. Hierzu trug 1991 die Gründung des AAOIFI

| 452 | (Accounting and Auditing Organization for Islamic Financial Institutions), eine der wichtigsten internationalen Organisationen zur Standardisierung im islamischen Finanzwesen, bei (Wilson, 2009, S. 26).

Diese ersten Jahrzehnte des islamischen Finanzwesens belegen, dass islamische Finanzinstitute im Golf auch in Zeiten niedriger Ölpreise in den 1980er und 1990er Jahren überlebensfähig waren, die 
Geschäftsmodelle weiterentwickelt wurden und den Zuspruch von Kunden fanden. Trotz des Wachstums dieser Branche blieb das islamische Finanzwesen mit Ausnahme in Iran jedoch eher ein Nischenmarkt.

\section{Die 2000er Jahre und die Auswirkungen der Globalen Finanzkrise}

Während der 2000er Jahre bis zur Globalen Finanzkrise wuchs das islamische Finanzwesen in den Golfstaaten und weltweit mit hohen Wachstumsraten. Dies war einerseits bedingt durch eine günstige demographische Entwicklung mit einer wachsenden jungen Bevölkerung und andererseits durch steigende Ölpreise, welche die Konjunktur in den Golfstaaten unterstützten (El-Ghattis, 2014, S. 30-31). Islamische Banken profitierten von der Nachfrage nach Konsumentenkrediten und Autokrediten. Sie entwickelten auch islamkonforme Kreditkarten, welche den Konsumgewohnheiten ihrer Kunden noch mehr entgegenkamen. Hinzu kam ein Boom im Bau- und Immobiliensektor. Die Bürger der GCC Staaten bauten und kauften Immobilien nicht nur für sich selbst, sondern auch als Investitionen, um diese an die zahlreichen Arbeitsimmigranten zu vermieten. Später kam Immobilienspekulation hinzu. Dieser Boom des Immobilienmarktes war speziell für die islamischen Finanzinstitute hilfreich, da islamische Finanzinstrumente eine Basis im realen Sektor benötigen. Die Entwicklung von innovativen islamischen Finanzinstrumenten erweiterte andererseits die Möglichkeiten des islamischen Finanzwesens. So ließen sich nun auch komplexe Großprojekte des Privatsektors und des Staatssektors islamkonform finanzieren. Hierzu trug nicht nur das islamische Bankwesen, sondern auch ein Boom von sukuk Emissionen bei. Im Allgemeinen besaßen die islamischen Banken aber für syndizierte Kredite für Großprojekte zu wenig Erfahrung und waren zu klein, so dass hierfür konventionelle, internationale Großbanken mit einbezogen wurden (Wilson, 2009, S. 16-21).

Zwischen den GCC Staaten bestanden Unterschiede in den Geschäftsmodellen. In Saudi-Arabien hielten viele Anleger ihr Geld auf Girokonten, welche keinerlei Vergütung gewährten. Die Gewinnsituation für saudische Banken wie Al-Rajhi war daher besonders günstig. Profitorientierte Anleger in Saudi-Arabien legten ihr Geld in islamkonformen Fonds an. Die Situation in den kleinen Golfstaaten unterschied sich hiervon. Auch hier gab es Anlagen in Girokonten ohne Vergütung, aber dies war ein kleinerer Teil. Die Mehrheit der Anlagen floss in Spar- und Investitionskonten, welche auf dem Modell der mudaraba beruhten und eine Gewinnbeteiligung versprachen (Wilson, 2009, S. 12-15).

Das dynamische Wachstum wurde durch die Globale Finanzkrise (2007-2009) zeitweise gestoppt. Zwar waren islamische Finanzinstitute aufgrund ihrer Prinzipien nicht in toxischen Wertpapieren, wie zinstragenden subprime asset-backed securities oder credit default swaps, investiert und waren somit nicht von der ersten Welle der Finanzkrise betroffen. Im Jahr 2008 konnten islamische Finanzinstitute daher höhere Profitraten als ihre konventionellen Konkurrenten aufweisen. Jedoch fielen die Kurse der sukuk schon in 2008 aufgrund der Liquiditätsengpässe der Investoren weltweit. Von der zweiten Welle der Finanzkrise, welche durch die realwirtschaftlichen Konsequenzen verursacht wurde, blieben auch die islamischen Finanzinstitute nicht verschont. So wiesen islamische Finanzinstitute in 2009 eine niedrigere Profitrate als ihre konventionellen Konkurrenten aus (Hasan \& Dridi, 2010, S. 15-16). Da sie im Immobiliensektor überinvestiert waren, welcher im Golf schon Symptome einer Blase entwickelt hatte, traf sie ein Fallen der Immobilienpreise besonders hart. Um Konkurse von islamischen 
Finanzinstituten zu verhindern, waren auch in der Golfregion staatliche Hilfen und Umstrukturierungen notwendig. Volker Nienhaus erwähnt hier als wichtige Beispiele The Investment Dar in Kuwait, Gulf Finance House in Bahrain (Khnifer et al., 2010), Tamweel und Amlak in Dubai sowie zwei islamische Banken in Katar (Nienhaus, 2011, S. 294).

\section{Gegenwärtige Lage, Chancen und Probleme}

Nach der Krise erholte sich das islamische Finanzwesen schnell. Gestützt wurde dieses Wachstum durch die Konjunktur in den Golfstaaten, die Präferenzen der Kunden und staatliche Unterstützung. So wuchsen die shari'a-konformen Anlagen in den GCC Staaten von schätzungsweise 263 Mrd. \$ in 2007 auf ca. 927 Mrd. \$ in 2018. ${ }^{3}$ Unter diesen Anlagen überwogen die islamischen Bankaktiva gefolgt von den islamischen Anleihen (sukuk). Islamische Fonds und islamische Versicherungen (takaful) spielen bis jetzt nur eine nachrangige Rolle (siehe Tabelle 1):

Tabelle 1: Islamisches Finanzwesen in den GCC Staaten in 2018, in Mrd. US-Dollar

\begin{tabular}{lc}
\hline Bankaktiva (Juni 2018) & 704,8 \\
\hline Ausstehende Sukuk (Ende 2018) & 187,9 \\
\hline Islamische Fondvermögen (Ende 2018) & 22,7 \\
\hline Takaful Beiträge (Ende 2017) & 11,7 \\
\hline
\end{tabular}

Quelle: Islamic Financial Services Board [IFSB], 2019, S.10.

In den meisten Ländern des arabisch-persischen Golfs hat sich das islamische Bankwesen zu einer systemrelevanten Größe für das nationale Bankwesen entwickelt. Das Islamic Financial Services Board (IFSB) beurteilt als systemrelevant, wenn das islamische Bankwesen 15\% oder mehr des Bankwesens eines Landes ausmacht, oder dessen islamische Banken 5\% oder mehr des islamischen Bankwesens weltweit beträgt. Nach diesem Kriterium ist das islamische Bankwesen in Iran, Saudi-Arabien, Kuwait, Katar und den Vereinigten Arabischen Emiraten (VAE) systemrelevant (siehe Tabelle 2):

Tabelle 2: Anteil des islamischen Bankwesens an den gesamten Bankaktiva in \% (2.Q. 2018)

\begin{tabular}{|c|c|c|}
\hline \multirow{7}{*}{ 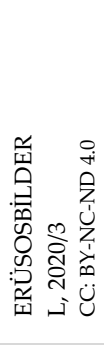 } & Iran & 100 \\
\hline & Saudi-Arabien & 51,5 \\
\hline & Kuwait & 40,6 \\
\hline & Katar & 25,2 \\
\hline & VAE & 20,6 \\
\hline & Bahrain & 14,3 \\
\hline & Oman & 12,4 \\
\hline
\end{tabular}

| 454 | Quelle: IFSB: 2019, S.10-11; 13.

\footnotetext{
${ }^{3}$ Die erste Zahl beruht auf Schätzungen von Wilson, 2009, S. 3-4 und die zweite auf Schätzungen des IFSB: Islamic financial services industry stability report 2019, S.10.
} 
Bahrain stellt einen Sonderfall dar. Seine islamischen Bankaktiva bleiben etwas unter $15 \%$ der gesamten Bankaktiva. Das Land möchte aber sowohl ein Zentrum des konventionellen wie des islamischen Finanzwesens in der Region sein. Daher befinden sich wichtige internationale Organisationen des islamischen Finanzwesens wie AAOIFI in Bahrain und in dieser Hinsicht kann das islamische Finanzwesen als systemrelevant für Bahrain gelten.

Oman hatte sich lange Zeit aus politischen Gründen gegen das islamische Finanzwesen gesperrt, da man fürchtete, dass es islamischen Extremismus fördern könne. Aber mit dem weltweiten Erfolg des islamischen Finanzwesens wurde deutlich, dass es sich zu einem normalen Geschäft entwickelt hatte. Die ablehnende Haltung in Oman änderte sich daher in $2011 \mathrm{im}$ Zuge des Arabischen Frühlings als ein Zugeständnis an die öffentliche Meinung. 2012 wurde das Bankwesen für islamische Banken geöffnet und Oman gründete ein Nationales Shari’a Board. Als erste islamische Banken betraten Al Izz Islamic Bank und Bank Nizwa den Markt, während konventionelle Banken „islamische Fenster“ eröffneten (Babicci \& Wongsurawat, 2020, S. 115-116, S. 120-122; Gulf News, 2014). Letztere sind keine unabhängigen Unternehmen, sondern Abteilungen der konventionellen Banken, welche shari'akonforme Produkte anbieten und deren Vermögen vom konventionellen Geschäft getrennt sind. Das islamische Bankwesen hat in Oman schnell an Boden gewonnen und erreichte 2018 einen Anteil von 12,4\% der gesamten Bankaktiva in Oman (IFSB, 2019, S. 10-11, S. 13).

Die islamischen Finanzinstitute der GCC Staaten verstärken ihr Engagement in anderen Weltregionen und sind hierbei aktiver als die konventionellen Finanzinstitute der GCC Staaten (Hanieh, 2019, S. 9). Insgesamt hat die Dominanz der Golfregion und hier vor allem der GCC Staaten im islamischen Finanzwesen zugenommen. Dies trifft besonders auf das islamische Bankwesen zu. Hier betrug der Anteil der GCC Staaten in 2012 schätzungsweise 34,1 \% und der des Iran in 2011 39,7\% (IFSB, 2013, S. 9 u. 12). Der Anteil der GGC Staaten stieg bis zum 1. Halbjahr 2018 auf 43,9\% und der des Irans fiel auf 32,1\%. (siehe Tabelle 3). Der Anteil der Golfregion insgesamt am islamischen Bankwesen hat also leicht von $73,82 \%$ auf $76,95 \%$ zugenommen.

Tabelle 3: Anteil der Staaten an den globalen islamischen Bankaktiva in \% (2.Q. 2018)

\begin{tabular}{ll}
\hline Iran & 32,1 \\
\hline Saudi-Arabien & 20,2 \\
\hline VAE & 9,8 \\
\hline Kuwait & 6,3 \\
\hline Katar & 6,2 \\
\hline Bahrain & 0,7 \\
\hline Oman & 0,7 \\
\hline
\end{tabular}

Quelle: IFSB, 2019, S.12.

\footnotetext{
${ }^{4}$ Im IFSB: Islamic financial services industry stability report 2019 gibt es eine Unstimmigkeit in Bezug auf diesen Anteil. Während die Länderanteile sich klar zu 43,9\% aufaddieren, wird an gleicher Stelle der Anteil der GCC Staaten am Bankwesen insgesamt mit 44,9\% angegeben. Für die Interpretation der Tendenz des Wachstums des Anteils der GCC seit 2012 spielt dieser Unterschied allerdings keine Rolle.
} 
Der Eindruck dieser führenden Rolle der GCC Staaten im islamischen Bankgeschäft verstärkt sich, wenn man ihre internationalen Verflechtungen betrachtet. In einer Studie von Adam Hanieh (2019) bewertet dieser das globale Gewicht der islamischen Banken des GCC. Hierfür werden islamische Banken als mit GCC Banken verbunden betrachtet, wenn GCC Banken 20\% oder mehr ihres Kapitals halten. Nach diesem Kriterium beherrschten islamische Banken aus den GCC Staaten und mit ihnen verbundene Banken mehr als 50\% der islamischen Bankaktiva in Ländern wie Ägypten, BosnienHerzegowina, Brunei, Dschibuti, Jordanien, Kasachstan, Pakistan, Senegal und der Türkei. Auch in anderen Ländern halten sie 30\% oder mehr. In Malaysia jedoch war ihr Anteil mit 9\% vergleichsweise gering. Im Allgemeinen steigt mit der Aktivität der islamischen GCC Banken auch der Einfluss und die Bedeutung der GCC Staaten auf andere Wirtschaftssektoren in diesen Ländern (Hanieh, 2019, S. 10-12).

Auch auf dem sukuk Markt haben die GCC Staaten an Bedeutung gewonnen. Während in 2012 die GCC Staaten einen Anteil von 28,9\% an den ausstehenden sukuk weltweit hatten und Malaysia die führende Rolle auf diesem Markt spielte (IFSB, 2013, S. 9 u. 26), hat sich der Anteil der GCC Staaten bis 2018 auf 35,4\% erhöht (IFSB, 2019, S. 10). Bei den Neuemissionen des Jahres 2018 war Malaysia wiederum mit 35,2\% das Land mit dem höchsten Anteil. Zusammen genommen emittierten die GCC Staaten aber 43,8\% der sukuk (siehe Tabelle 4). In diesem Markt spielen Südostasien und hier insbesondere Malaysia also weiterhin eine wichtige Rolle. Das Gewicht ändert sich aber langsam zugunsten der GCC Staaten. Dies hat unter anderem damit zu tun, dass mit dem Sinken der Ölpreise seit Mitte 2014 GCC Staaten verstärkt die Emission von sukuk zur Staatsfinanzierung nutzten. GCC Banken waren auch als Arrangeure und Berater für sukuk Emissionen tätig und der Börsenplatz Dubai hat sich zu einem Zentrum für die Notierung und den Handel von sukuk entwickelt (Hanieh, 2019, S. 12-15). Zwischen Malaysia und den arabischen Golfstaaten bestehen unterschiedliche Rechtsauffassungen über den Handel mit islamkonform entstandenen Schulden zu einem anderen Preis als dem Nennwert. In Malaysia wird dies als erlaubt anerkannt und entsprechende islamkonforme Wertpapiere angeboten. In den Golfstaaten gilt dies aber als riba. Im Laufe der Zeit ist zu erwarten, dass die Auffassung der arabischen Golfstaaten die größere Anerkennung finden wird, insofern potentielle Emittenten von sukuk die Investoren aus den Golfstaaten ansprechen wollen.

Tabelle 4: Anteil an den weltweiten sukuk Emissionen im Jahr 2018 in \%

\begin{tabular}{|c|c|c|}
\hline \multirow{5}{*}{ 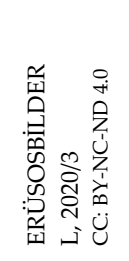 } & Malaysia & 35,2 \\
\hline & Saudi-Arabien & 23,1 \\
\hline & VAE & 13,1 \\
\hline & Indonesien & 11,2 \\
\hline & Türkei & 8,3 \\
\hline \multirow[t]{3}{*}{ | 456 | } & Oman & 3,7 \\
\hline & Bahrain & 2,7 \\
\hline & Katar & 1,2 \\
\hline
\end{tabular}

Quelle: IFSB, 2019, S.20. 
Die Sonderrolle des Iran im islamischen Finanzwesen hat sich im Wesentlichen nicht geändert. Einerseits besitzt der Iran im internationalen Vergleich das größte islamische Bankwesen, welches 2018 einen Anteil von 32,1\% an den islamischen Bankanlagen weltweit ausmachte. Andererseits ist es durch die Sanktionen der USA und durch eine unterschiedliche Interpretation der islamischen Finanzinstrumente von den Entwicklungen auf den anderen islamischen Finanzmärkten isoliert. Zwar wurde zwischenzeitlich seit 1999 das iranische Bankwesen wieder für den Privatsektor geöffnet, jedoch wird es immer noch vom Staat dominiert. Dies führt zu Ineffizienz, welche sich in einem hohen Anteil von notleidenden Darlehen äußert (Vizcaino, 2015). Während an sich ein hohes Potential für eine Modernisierung des islamischen Finanzwesens durch eine verstärkte Zusammenarbeit mit anderen Zentren des islamischen Finanzwesens bestünde, ist dies aufgrund der internationalen Spannungen zwischen dem Iran und den USA, wie auch zwischen dem Iran und Saudi-Arabien für die nahe Zukunft nicht zu erwarten.

\section{Ausblick}

Seit der Gründung der ersten kommerziellen islamischen Banken haben sich Akteure aus den Golfstaaten um die Verbreitung des islamischen Finanzwesens nicht nur in der eigenen Region, sondern auch in anderen Regionen bemüht. Inzwischen ist das islamische Finanzwesen eine anerkannte, weltweit verbreitete Industrie geworden. Interessanterweise hat diese Verbreitung nicht zu einem abnehmenden Anteil der Golfregion im islamischen Finanzwesen geführt. Im Gegenteil, dieser Anteil hat in den vergangenen Jahren noch zugenommen. Hierbei spielt einerseits ein zunehmender Marktanteil islamischer Banken in den eigenen Ländern eine Rolle und andererseits ihre aktive Rolle in der Internationalisierung des islamischen Bankwesens. Auch im sukuk Markt konnten die GCC Staaten im Vergleich zu Malaysia an Bedeutung gewinnen. Die Golfregion ist daher zum Zentrum des islamischen Finanzwesens und das islamische Finanzwesen $\mathrm{zu}$ einem ökonomischen Charakteristikum der Golfregion geworden.

Dieser Erfolg beruht auf der Präferenz weiter Teile der Bevölkerung für islamkonforme Finanzprodukte und die Finanzkraft der Ölstaaten. Allerdings belegt das langfristige Wachstum des islamischen Finanzwesens in der Golfregion, dass es auch in Phasen niedriger Ölpreise überlebensfähig ist und seinen Anteil ausbauen kann. Die langfristige politische Unterstützung der unterschiedlichen Regime der Golfstaaten ist ein weiterer wichtiger Erfolgsfaktor. Zugleich beschränken die politischen Interessen auch das islamische Finanzwesen, wie die Isolation Irans und auch die politischen Spannungen zwischen Katar einerseits und Saudi-Arabien, Bahrain und den VAE andererseits belegen.

Da islamische Banken in einigen GCC Staaten schon sehr hohe Marktanteile erreicht haben, wird ein weiteres Wachstum auf diesem Wege schwieriger werden. Das Augenmerk könnte in Zukunft daher mehr auf der Entwicklung weiterer innovativer islamkonformer Finanzinstrumente und einer zunehmenden internationalen Standardisierung des islamischen Finanzwesens liegen, da bis jetzt erhebliche Unterschiede zwischen den nationalen Regulierungen bestehen.

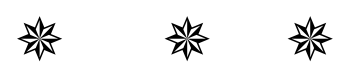




\section{LITERATURVERZEICHNIS}

Babicci, K., \& Wongsurawat, W. (2020). Islamic banking in Oman: Laying the foundations. Middle East Policy, 27(1), 115-124.

Brauksiepe, R. (1992). Das Bankwesen der Islamischen Republik Iran: Konzepte, Erfahrungen und Perspektiven. Materialien und Kleine Schriften 138, Institut für Entwicklungsforschung und Entwicklungspolitik der Ruhr-Universität Bochum.

El-Ghattis, N. (2014). The futures of Islamic banking in the Gulf Cooperation Council (GCC). Journal of Future Studies, 18(4), 27-44.

Hanieh, A. (2019). New geographies of financial power: Global Islamic finance and the Gulf. Third World Quarterly, 1-22, https://doi.org/10.1080/01436597.2019.1675505

Hasan, M., \& Dridi, J. (2010). The effects of the global crisis on Islamic and conventional banks: A comparative study. IMF Working Paper WP/10/201, Washington D.C.: International Monetary Fund. https://www.imf.org/ /media/Websites/IMF/imported-full-textpdf/external/pubs/ft/wp/2010/_wp10201.ashx

Henry, C. M. (1997). The Mediterranean debt crescent: Money and power in Algeria, Egypt, Morocco, Tunisia, and Turkey. The American University in Cairo Press.

Henry, C. M. (2005). Financial performances of Islamic versus conventional banks. In C. M. Henry \& R. Wilson (Eds.), The politics of Islamic finance (pp. 104-128). First published by Edinburgh University Press 2004, Reprint in Karachi: Oxford University Press, 2005.

Islamic Financial Services Board (IFSB) (2013). Islamic financial services industry stability report 2013. Kuala Lumpur: Islamic Financial Services Board. https://www.ifsb.org/download.php?id=4415\&lang=English\&pg=/sec03.php

Islamic Financial Services Board (IFSB) (2019). Islamic financial services industry stability report 2019. Kuala Lumpur: Islamic Financial Services Board. https://www.ifsb.org/download.php?id=5231\&lang=English\&pg=/sec03.php

Kahf, M. (2005). Islamic banks: The rise of a new power alliance of wealth and shari'a scholarship. In C. M. Henry \& R. Wilson (Eds.), The politics of Islamic finance (pp. 17-36). First published in Edinburgh: Edinburgh University Press 2004, Reprint in Karachi: Oxford University Press, 2005.

Khnifer, M., Baig, A., \& Winkler, F. (2010). The rise and fall of Gulf Finance House. https://ssrn.com/abstract=1712059 oder http://dx.doi.org/10.2139/ssrn.1712059

Nienhaus, V. (1982). Islam und Moderne Wirtschaft: Einführung in Positionen, Probleme und Perspektiven, Styria.

Nienhaus, V. (2011). Islamic finance ethics and sharia'ah law in the aftermath of the crisis: Concept and practice of shari'ah compliant finance. Ethical Perspectives, 18(4), 591-623.

Obaidullah, M. (2005). Islamic financial services. King Abdulaziz University.

Gulf News (2014, 08. Oktober). Oman sets up central sharia board to boost Islamic finance. https://gulfnews.com/business/oman-sets-up-central-sharia-board-in-move-to-boost-islamicfinance-1.1396051 
Valibeigi, M. (1992). Banking and credit rationing under the Islamic Republic of Iran. Iranian Studies, 25(3/4), 51-65.

Vizcaino, B. (2015, 12. Mai). Iran's isolated banks may have slow, painful return to global system, Reuters. https://in.reuters.com/article/us-iran-banks/irans-isolated-banks-may-have-slow-painfulreturn-to-global-system-idINKBN0NX0VK20150512

Warde, I. (2000). Islamic finance in the global environment. Edinburgh University Press.

Wilson, R. (1983). Banking and finance in the Arab Middle East, MacMillan Publishers.

Wilson, R. (2009). The development of Islamic finance in the GCC, Kuwait Programme on Development, Governance and Globalisation in the Gulf States. London School of Economics and Political Science.

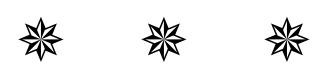

